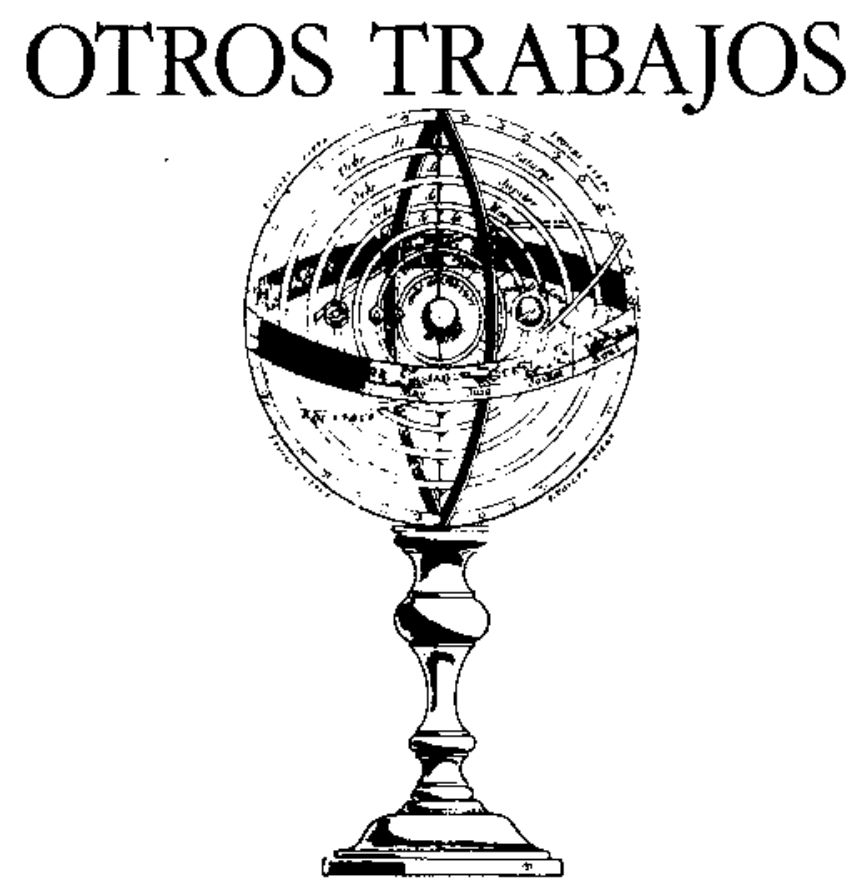

\title{
¿QUÉ PUEDEN APORTAR A LOS ENSEÑANTES LOS DIFERENTES ENFOQUES DE LA DIDÁCTICA DE LAS MATEMÁTICAS? (Primera parte)
}

\section{BROUSSEAU, G.}

IREM, Université de Bordeaux I, Francia.

Versión castellana de Luis Puig.

\section{SUMMARY}

This paper offers a general view of the relationships between Education, Mathematics Education and Mathematics.

\section{ACEPCIONES DE LA PALABRA 'DIDACTICA'}

En su empleo más corriente, 'didáctica' es un adjetivo, califica «lo que es apropiado para la enseñanza, to que ENSEÑANZA DE LAS CIENCIAS, 1990, 8 (3), 259-267 tiene por finaliđad la enseñanza y, más en general, lo que está relacionado con la enseñanza». 
Jan Amos Komensky, llamado Comenius, la introdujo como substantivo en 1640 para designar «el arte de enseñar», es decir, el conjunto de medios y procedimientos que tienden a hacer conocer, a hacer saber algo, generalmente una ciencia, una lengua, un arte... Este sentido original es el más extendido.

Algunas instituciones que, por razones diversas, quieren colocar la práctica de la enseñanza a una cierta distancia, utilizan este término un poco afectado según sus necesidades. Inmediatamente aparecen tres direcciones:

1. 'Didáctica' sería una palabra culta para designar la enseñanza.

\section{La didáctica sería la preparación de lo que sirve para} enseñar.

\section{La didáctica sería el conocimiento del arte del ense-} ñar.

1.1. La primera acepción parece justificada ya que en tanto práctica social la enseñanza es por excelencia el medio institucional del que se dota la sociedad para hacer conocer el saber, la cultura, a todos sus miembros. $\mathrm{El}$ acto de enseñar puede ser interpretado, por tanto, como el ejercício mismo de la didáctica. Afinando la definición de Comenius, la didáctica se convierte en ei proyecto social de hacer apropiar -por un alumno o varios - un saber constituido o en vias de constitución.

Esta definición pone el acento sobre el papel del saber - no compete a la didáctica más que lo que, en el proyecto educativo, es específico del saber que se tiene en el punto de mira-; sobre el carácter social del proyecto y, por tanto, sobre las posibilidades culturales de identificación y de gestión de los saberes; y sobre los protagonistas y sobre el carácter institucional de la acción (incluso el autodidacta reatiza un proyecto social).

1.2. La segunda acepción se apoya más bien sobre el sentido del adjetivo: la didáctica es pues el conjunto de las técnicas que sirven para enseñar, en oposición al propio acto de enseñar. Designa, entonces, una actividad original: «la invención, la descripción, el estudio, la producción, la difusión y el control de nuevos medios para la enseñanza: currículos, objetivos, medios de evaIuación, materiales, manuales, software educativo, obras de formación...». Este significado está muy extendido en Canadá y un poco menos en Estados Unidos, pero existen términos en diversos países que tienen un significado próximo: la pedagogía especial, que tiende a derivar estas técnicas de principios educativos más generales, o la metodología, que parece más independiente.

1.3. En oposición a las dos primeras acepciones, que hacen de la diđáctica un conjunto de datos más bien normativos, prescriptivos y organizados para la decisión y la acción, la tercera hará de ella por el contrario la descripción y el estudio de la actividad de enseñanza en el marco de una disciplina científica de referencia. Se trata, en este caso, de una posición mucho más reflexiva: la didáctica, conocimiento del arte de enseñar, se convierte en un campo de investigación.
En el primer sentido, el didacta es un enseñante que hace esfuerzos particulares para determinar el objeto y los métodos de su enseñanza. En el segundo, es un técnico o un ingeniero, que produce y propaga innovaciones. En el tercero, es un investigador, que se distingue en su disciplina porque su objeto de estudio tiene que ver con la enseñanza.

Señalemos aquí que la cultura atribuye a la palabra 'didáctica' otro significado muy peyorativo: en francés, 'didáctica' se usa habitualmente como sinónimo de 'pedante' y de 'pretencioso'. Por supuesto, un objeto es «didáctico» cuando deja que se trasluzca la intención de enseñar, es, por tanto, un objeto que ha sido desviado de su función natural hacia fines de enseñanza: una poesía didáctica, un tono didáctico, un material didáctico (por ejemplo, un motor cortado para mostrar su interior) ya no son adecuados para su función primera, por lo que, fuera del campo escolar adquieren un carácter ineficaz y riđículo. Pero esta explicación no basta: el objeto no se toma más que como un medio de ridiculizar la propia intención de enseñar y lo que se pretende es ridiculizar el intento de considerar seriamente el conocimiento de los medios de enseñar. Habrá que intentar comprender por qué.

En este punto se podría creer que el trabajo teórico está terminado: está claro que estos diversos usos de la palabra 'didáctica' son perfectamente compatibles e, incluso, en cierta forma complementarios. Bastaría pues con ilustrar cada uno de esos enfoques mediante sus mejores realizaciones: las innovaciones, las técnicas para la enseñanza, los conocimientos sobre la enserianza.

1.4. Pero desde hace una quincena de años ha aparecido, también bajo el nombre de 'didáctica', un intento de constituir una ciencia de la comunicación de los conocimientos y de sus transformaciones; una epistemología experimental que intenta teorizar la producción y la circulación de los saberes un poco como la economía estudia la producción y la distribución de los bienes materiales. Esta ciencia se interesa, en lo que estos fenómenos tienen de específico del conocimiento que se tiene en el punto de mira, por la manera como conocimientos escasos se usan para la satisfacción de las necesidades de los hombres que viven en una sociedad y, en particular, por las operaciones esenciales de la difusión de los conocimientos, las condiciones de esta difusión y las transformaciones que esta difusión produce, tanto sobre esos conocimientos como sobre sus usuarios; por las instituciones y las actividades que tienen como objeto facilitar esas operaciones.

Este enfoque científico y unitario puede aparecer como el resultado de exigencias excesivas, pretenciosas y superfluas. ¿Es necesaria una teorizacion tal? ¿Ofrece a los enseñantes apoyos originales, más sólidos, más eficaces y más útiles? ¿No sería preferible un sólido pragmatismo?

Ciertamente, el programa de trabajo propuesto por las posiciones clásicas parece irremediablemente enorme y fundamentalmente desordenado. Casi ninguna de las declaraciones sobre la enseñanza parece efectivamente 
falsabie ${ }^{(3)}$. Como consecuencia de ello, deja a los enseñantes al cuidado de la integración de todas las aportaciones $\mathrm{y}$, por ello, a los ensefiantes les incumbe lo esencial de las responsabilidades. Una teorización específica permitiría la coordinación y el análisis de las aportaciones de los otros sectores científicos y un mejor reparto de tareas. La teorización de la enseñanza es pues una tentación permanente y seductora. Pero, ¿cuál es la legitimidad de una teoría aparentemente «home made»?, ¿permitirá al menos engranar la dialéctica científica?

Vamos a intentar contestar a estas preguntas. Para ello, una panorámica previa del campo y de los conceptos de la didáctica es indispensable. Estudiaremos a continuación las aportaciones y los límites de los enfoques clásicos, aplicándoles los métodos de análisis que esta teorización propone. Volveremos luego sobre una parte esencial de nuestro propósito: las relaciones de la didáctica de Ias matemáticas con las propias matemáticas; ¿permitirá la diđáctica mejorar de forma fundamental la difusión en la cultura común del pensamiento matemático?, ¿lo hará salir de su gueto?

\section{INTRODUCCIÓN A LA DIDÁCTICA: ¿QUÉ PUEDE APORTAR A LOS ENSENANTES?}

La idea fundamental consiste en postular que cada conocimiento o cada saber debe poder ser determinado por una situación. Una situación es un conjunto de relaciones que ligan a un agente o a varios. Estas relaciones deben ser tales que ese conocimiento es necesario para su realización o para su mantenimiento, por ejemplo, esas relaciones pueden ser un juego en el que la puesta en funcionamiento del conocimiento en cuestión es el único medio de asegurar al jugador una estrategia ganadora óptima. Está claro que no se ha inventado y no se utiliza el teorema de Pitágoras y el teorema de Varigon con el mismo tipo de relación con el medio.

Los conocimientos pueden aparecer en situaciones originales, pero los saberes culturales están asociados necesariamente a prácticas sociales que les sirven de referencia.

Un corolario del postulado fundamental es que a situaciones diferentes les corresponden conocimientos diferentes. Por consiguiente, el saber nunca es exactamente el mismo para sus creadores, para sus usuarios, para los alumnos, etc., cambia. El estudio y el control de estas modificaciones, que nosotros llamamos transposición didáctica es el objeto principal de Ia teoría. Por tanto, to único que se puede hacer es, en el mejor de Ios casos, modelizar las situaciones características de un saber, pero ya se dibuja una primera aportación de la diđáctica.

\subsection{Primera aportación: técnicas para el enseñante}

2.1.1.Los profesores esperan que, por lomenos, la didáctica les proporcione lo esencial de las técnicas espefícicas de las nociones que hay que enseñar, compatibles con sus con- cepciones educativas y pedagógicas generales.

-Técnicas locales

-Comunes: preparación de lecciones, materiales de enseñanza, métodos listos para usar, instrumentos de gestión, objetivos y evaluaciones.

-Especiales, para ciertos alumnos que presentan dificultades particulares.

-Técnicas globales, currículos para todo un sector de las matemáticas, programas para varios años.

Esta expectativa es legítima: los didactas han comenzado a estudiar numerosas situaciones de enseñanza, originales o no, sobre todo en el nivel elemental o en el nivel superior. Pero estos estudios son largos y difíciles.

Por ejemplo, en la introducción del álgebra, todo, o casi todo, está por hacer, incluso si algunas de las vías posibles empiezan a ser exploradas: ¿puede introducirse hoy en día el álgebra como reanudación teórica del estudio de las aritmética y los números?, ¿o como un sistema de designación de magnitudes?, ¿o como instrumento del estudio de las funciones?, 20 como sistema formal autónomo?, ¿o como taquigrafía de algoritmos que se refieren a valores desconocidos o no determinados?, to como medio de generalización o de modelización?... Se pueden improvisar montajes diversos, pero, antes de poder proponer uno cualquiera, hay que examinar sus propiedades con respecto a un número considerable de exigencias. Como señalaba Dieudonné «mientras que no hizo falta mucho más de un siglo para que la geometría elemental alcanzara una forma casi definitiva, sólo trece siglos después de Diofanto el álgebra llegó a ser lo que conocemos», de modo que el paso no debe de ser tan evidente.

Además, el número de conocimientos que hay que comunicar a los alumnos y, por tanto, el de situaciones específicas que hay que proponerles es muy elevado. Para ser razonablemente comunicable a los enseñantes, la didáctica debe, por tanto, producir también conceptos unificadores, reagrupar los saberes, los problemas, las situaciones, los comportamientos de los alumnos o las actividades, de manera que se puedan enunciar formas de intervención genéricas, según los tipos obtenidos.

La existencia de una técnica se apoya al menos sobre la identifícación y el reconocimiento de las prácticas y de sus resultados canónicos. Una ingeniería apoya sobre un campo científico las técnicas que propone. La comunicación, la utilización y la reproducción de las situaciones producidas requiere el recurso a conocimientos y saberes específicos.

Así, la didáctica es el único medio de localizar exactamente lo que es un problema no resuelto de ingeniería didáctica, de identificar y de clasificar un trabajo original en este dominio, de precisar sus condiciones de uso y de reproducción, $y$, por tanto, de reconocer las creaciones, las invenciones y los procesos de búsqueda y de producción cientffica que realizan los enseñantes, así como de hacer que todo ello se reconozca. 
Al definir y al hacer respetar la parte técnica del oficio del profesor, la didáctica hace posible la negociación social de su trabajo. Es así el fundamento de la profesionalización de su actividad.

Pero el hecho de hacer un catálogo de las situaciones de enseñanza no les da ninguna virtud para la enseñanza. ¿Qué ventajas podrían tener para los niños?

Los ejemplos a los que puede uno referirse muestran que las situaciones «buenas», las que permiten que se realicen condiciones pedagógicas más exigentes, es decir, lecciones más seguras para el profesor a la vez que más abiertas para el alumno, sólo pueden comunicarse si han sido bien estudiadas. La didáctica ha introducido situaciones muy variadas y mejor adaptadas a intenciones específicas, como, por ejempio, hacer que se adquiera un conocimiento mediante la acción, hacer que se adquiera un lenguaje, o una teoría, tratar un obstáculo epistemológico...

2.1.2. Se me perdonará que recuerde aquí un ejemplo personal, pero bien conocido no sólo por los lectores franceses', sino también por los españoles gracias al libro de Julia Centeno (1988).

Indiquemos brevemente su contexto. Se trata, después de haber estudiado Ios números racionales y los decimales en tanto que medidas ( $1.78 \mathrm{~m}$, por ejemplo), de hacer extender su uso por los alumnos a la designación y al cálculo de las aplicaciones lineales (multiplicar por 1.78 , por ejemplo). Los estudios mostraban que los métodos clásicos de introducción, basados, en su mayor parte, sobre la construcción explícita por composición de las aplicaciones naturales ya conocidas por los alumnos (multiplicar por $178 \mathrm{y}$ dividir por 100), presentaban insuficiencias. La idea consistía en colocar a los alumnos ante la obligación de usar implícitamente tales aplicaciones (una familia bastante numerosa) y de prever sus efectos y sus propiedades (ordenarlas, prever su suma, su producto...), de manera que se vean conducidos a buscar una manera cómoda de designarlas. Era preciso, pues, que aparecieran (en un primer momento una sola, luego otra y otras más) antes que nada como solución implícita de un problema de acción, con el fin de plantear a continuación los problemas de comunicación que justificarían explícitamente la búsqueda de un sistema de designación.

Tal problema de acción, recordémoslo, consiste para los alumnos en encontrar el modo de agrandar las piezas del puzzle, de tal manera que una pieza de lado 4 tenga como imagen una pieza de lado 7 y que el puzzle-imagen funcione como un auténtico puzzle y funcione bien.

La astucia consiste en pedir a grupos pequeños (de dos alumnos, para favorecer la expresión de los conflictos cognitivos que van a surgir) que cada uno reproduzca uno de los trozos del puzzle (para favorecer los conflictos que nacen de los intentos de ajuste de las piezas). A los alumnos les parece claro que hace falta que la imagen de cada longitud se calcule con operaciones aritméticas elementales, ¿pero cuáles?, $i$ «la longitud medida $+3 \mathrm{~cm} » ?, i$ «dos veces la longitud menos $1 »$ ?
El intento de acoplar las piezas constituye una retroalimentación real que sólo deja lugar para aplicaciones bastante cercanas a 4/7: "dos veces la longitud menos 1" funciona casi bien, excepto para $0.5 \mathrm{~cm}$ por supuesto.

La búsqueda por los alumnos de una solución intelectualmente satisfactoria va a ser la fuente de la comprensión, y, luego, de que se haga explícita la propiedad fundamental de la linealidad: es preciso que la imagen de la suma de dos longitudes sea la suma de las imágenes de esas longitudes. Pero si la solución no aparece demasiado pronto, la aplicación $X=4 / 7$ va a colocarse en un entorno implícito de funciones vecinas, en el sentido topológico, que prefigura la estructura estudiada un poco más tarde ${ }^{2}$.

El éxito de este proceso, experimentado en el $C M 2^{(i 1)}$ en condiciones favorables, ha dado mucha esperanza y entusiasmo, mostrando al mismo tiempo hasta qué punto su generalización a todas las clases de 6 ème $e^{(111)}$ sería difícil ${ }^{3}$.

2.1.3. Estas esperanzas de los profesores en la ingeniería son legítimas, pero se acompañan de presupuestos que lo son algo menos: haría falta que, para su puesta en práctica, estas técnicas no exigierán otros conocimientos ni otras condiciones que aquéllas de las que disponen actualmente los profesores, los alumnos y sus padres. $Y Y$ haría falta que su utilidad y su eficacia se revelaran, sin embargo, inmediatamente a los ojos de todos, bajo la forma de ventajas deslumbrantes y regulares al compararlas con las prácticas actuales!

Ahora bien, estas prácticas se consideran como harto conocidas, pero no están descritas en absoluto: cl número de parámetros de los que parecen depender las hace aparecer como muy poco homogéneas y, en consecuencia, pueden, según el momento, tanto ser cargadas de todas las virtudes como de todos los defectos.

Es muy difícil para un investigador o para un profesor publicar para sus colegas la descripción detallada de las condiciones necesarias para una situación de enseñanza (en el estado actual de la didáctica, esta descripción exige al menos una treintena de páginas), y le es igualmente difícil leer tal descripción escrita por sus colegas. La costumbre le conduce a no describir de ella más que su desarrollo y sus efectos, y esto de manera sucinta, Io que es totalmente insuficiente para una buena reproducción (sin embargo, basta leer las revistas y las obras en que se relatan lecciones para ver hasta qué punto estos rejatos pueden ser vagos, y compararlos con los corrientes hace una quincena de años para constatur los progresos realizados). ¿Puede alguien pensar que basta con enumerar las jugadas de una partida de maestros de ajedrez para que aparezcan sus estrategias y para que se pueda «reproducir» una partida ganadora? Mientras que la descripción exige esfuerzos considerables, todas estas precisiones aparecen como superfluas e incluso ofensivas para el lector, se reciben como un discurso pedante.

Los términos usados en esas descripciones deberían ser los que los profesores emplean en su trabajo. Pero si es indispensable utilizar conceptos que difieren de los que 
los profesores utilizan para designar objetos vecinos, aunque sólo sea porque se quiere controlar su definición teórica, entonces aparece el dilema siguiente: por una parte, el uso del término nuevo y alejado parece muy costoso; por otra parte, el uso del término corriente introduce contrasentidos y hace que las hipótesis o los resultados más seguros suenen como evidencias rimbombantes -se cree saber, ya que se conoce-; finalmente, la introducción del término evocador, pero original, despierta con razón la desconfianza y la ironía cuando se puede oponer una palabra familiar a la jerga.

En resumen, los profesores esperan la didáctica en un terreno en el que reinan como «amos» ${ }^{(\text {Iv) }}$ y en el que nada les prepara ni les motiva para recibirla tal como es.

\subsection{Segunda aportación: conocimientos sobre la en- señanza}

2.2.1. Los profesores pueden esperar también que la didáctica se manifieste mediante conocimientos relativos a aspectos diferentes de su trabajo:

-Sobre los alumnos, sus comportamientos, stus resultados en las condiciones específicas de la enseñanza.

-Sobre las condiciones que hay que crear en las situaciones de enseñanza y de aprendizaje.

-Sobre las condiciones que hay que mantener en la gestión o la conducción de la enseñanza.

-Sobre los fenómenos de didáctica a los que se enfrentan, tanto ellos como todos los que intervienen en la comunicación de saberes.

Sobre el primer punto es, sin ninguna duda, sobre el que hay resultados más numerosos y más seguros. Por otra parte, los profesores siempre están interesados por los informes en los que se exponen los errores y los comportamientos de los alumnos, pero queda mucho por hacer para comprender las causas de esos errores y no limitarse a constatarlos. Miremos, sin embargo, desde más cerca cómo esos tipos de conocimientos pueden intervenir en el tratamiento de un problema corriente de enseñanza.

Pongamos, no obstante, en guardia al lector: no se trata aquí, como se hace demasiado a menudo, de intentar exportar a priori la pregunta a una disciplina de referencia como la psicología, la neurofisiología o las matemáticas, para, de inmediato, volver a importar aplicaciones de ella, que también se impondrían con marchamo de calidad, sin que nunca se examine realmente la pertinencia del resultado. La mayor parte de las veces este proceso es simplemente una huida ante la necesidad de hacerse cargo del objeto didáctico que hay que estudiar. No está nada claro, por ejemplo, que el avance actual de las teorías de la memoria o del aprendizaje nos permita conocer sus límites de uso en la interpretacion de las cuestiones de enseñanza. Algunos de los resultados de la investigación en didáctica, tales como la puesta en evidencia de la obsolescencia de las situaciones didácticas y de los saberes, la del papel de los obstáculos epistemo- lógicos o del olvido, deberían bastar para ponernos en guardia contra estos abusos. Vamos a estudiar en un ejemplo el funcionamiento del análisis en diđáctica para mostrar de qué manera la didáctica plantea su problemátíca, cómo a veces responde a ella y en qué se opone al uso demasiado «ingenuo» (no a otros) de los conocimientos científicos.

\subsubsection{Ejemplo de preguntas}

En la escuela elemental, los alumnos se entrenan, al seguir las reglas del cálculo, en pasar de un término (sin variable) a otro -que le es igual-hasta la obtención del resultado en su forma canónica. $\ll 3+4=7 »$ se lee de la siguiente manera: «efectuando correctamente el cálculo $3+4$ se obtiene $7 » .3+4$ puede ser igual a 7 , pero no puede reemplazarlo como respuesta, no le es equivalente.

Cuando estos alumnos aprenden álgebra, se trata de entrenarse en pasar de una fórmula a otra que le es lógicamente equivalente, hasta la obtención de una relación utilizable para lo que se quiere hacer. $« 3+4=7$ y $7=4+x »$ implica $\langle x=3 \%$.

Superficialmente, se podría creer que profesor y alumno continúan haciendo uso de los mismos conocimientos antiguos, a los que vienen a añadirse otros nuevos; que se limitan a escribir lo que anteriormente sólo se pensaba; que bastaría con ver « $3+4$ » como un número, « $x »$ como un número desconocido, « $\# »$ como la identidad... De hecho, es harto conocido que todo ha cambiado acerca de esas escrituras familiares: el uso que se hace de ellas, el sentido que se les da, el objetivo de las transformaciones con las que se opera... El lector al que esta introducción ingenua y lapidaria decepcione puede remitirse con provecho a los trabajos de Yves Chevaliard sobre estas cuestiones (1989).

El alumno debe, por tanto, no sólo aprender conocimientos nuevos, sino también volver a aprender y volver a organizar los antiguos y olvidar -0 , mejor, desaprenderuna parte de ellos.

¿En qué medida es compatible esta observación con las bases de la evaluación, tal y como se practica hoy por hoy? ¿Permanecen válidas esas bases? ¿Podemos mejorarlas directamente sin implicar los métodos didacticos y las concepciones de los enseñantes? No sabemos distinguir bien las diferentes relaciones que el enseñante y sus alumnos pueden tener con un mismo conocimiento, ni describir las significaciones diferentes que un conocimiento puede tomar según las circunstancias en las que sirve y según la persona que se sirve de él.

No sabemos encarar bien el aprendizaje en términos de cambios de relaciones con el saber o en términos de transformaciones de los conocimientos del alumno. No sabemos describir bien el papel de los conocimientos antiguos en la construcción de los conocimientos nuevos.

¿Qué lugar hay que dejar en la enseñanza a estas reorganizaciones de conocimientos antiguos en relación con las yuxtaposiciones de aprendizajes nuevos? ¿Depende este lugar de las nociones? ¿Existen conocimientos que 
obstaculizan aprendizajes ulteriores? ¿Existen técnicas didácticas más favorables que otras, respecto a este asunto?

Actualmente, la integración de los conocimientos nuevos con los antiguos se deja totalmente a cargo del alumno, contentándose el profesor con comunicar por etapas trozos del saber "verdadero» de nuestra época. ¿Puede el enseñante escotomizar el pasado del niño dándole a entender que, excepto los algoritmos, todo lo que ha aprendido con anterioridad es inutilizable? iPuede comprender el alumno lo que se le enseña en esas condiciones?

Los currículos construidos hasta hoy no prevén nada que no sea la yuxtaposición de los aprendizajes: ¿qué consecuencias observables se pueden deducir de esta «insuficiencia» sobre la enseñanza y sobre los pasos de un nivel escolar a otro? ¿Y sobre las concepciones didácticas y epistemológicas de los profesores? ¿Se producen realmente tales consecuencias? ¿Sólo pueden ser imputadas a esta causa?

Por supuesto que sería absurdo concluir de ello que no hay que enseñar el cálculo en la escuela primaria y por supuesto que no resulta que sea fácil enseñar directamente elálgebra en laescuela primaria: la enseñanzadirecta del saber definitivo es imposible 0 , si se hace así, hay que renunciar a hacerlo funcionar. El uso y la destrucción de los conocimientos precedentes forman parte, por tanto, del acto de aprender.

En consecuencia, hay que admitir una cierta "reorganización diđáctica» del saber, que cambia su sentido, y hay que admitir, al menos a título transitorio, una cierta dosis de errores y contrasentidos, no sólo del lado de los alumnos, sino también del de la enseñanza.

Pero, $i$ cómo transformar el saber para convertirlo provisionalmente en inteligible, sin hacerlo demasiado falso ya que quedarán huellas que no podrán ser borradas...? ¿Y cómo rectificar a continuación esos errores?

¿Y de qué derecho podría arrogarse un profesor para hacer sufrir transposiciones didácticas al saber cultural común? ¿Cómo regular las distorsiones inevitables? ¿Puede estar esta tarea totalmente a cargo de un profesor o incluso de los profesores? ¿Se les puede imponer que enseñen conocimientos falsos, incluso provisionalmente, sin un acuerdo cultural a ese respecto? ise puede conseguir tal acuerdo si cada uno de los protagonistas se ve conducido a tener que ignorar cualquier análisis serio? ¿Quién se encarga de esta transacción, qué organización social puede permitirla en condiciones honestas para todos?

Éstas son algunas de Las preguntas «sencillas», casi ingenuas, que se plantean en didáctica de las matemáticas a propósito de un fenómeno corriente que compete a su campo.

A propósito de este fenómeno, salta a la vista una primera característica: se trata de la complejidad; complejidad que, en dominios muy variados, requiere tanto investigaciones experimentales y reflexiones sobre fun- damentos como invenciones o investigaciones de ingeniería. Esta complejidad se nota cuando se trata de plantear las preguntas necesarias para las investigaciones, pero llega a ser abrumadora cuando, con vistas a una decisión cualquiera, se trata de integrar las respuestas obtenidas eventualmente. Esta complejidad basta para justificar la desconfianza: se podría invertir etemamente en investigaciones de detalle sobre la enseñanza, sin que se obtuviera a cambio nada más que sugerencias dispersas y gratuitas.

Segunda característica importante: las preguntas planteadas requieren investigaciones en dominios de conocimientos muy diferentes, pero lo hacen de una manera que no parece dejar a ninguna de ellas la posibilidad de responder independientemente de las otras. Esta observación subraya la necesidad de un enfoque unitario y sistémico de las preguntas de didáctica.

\subsubsection{Ejemplos de procesos}

Continuemos el estudio de los cambios de niveles escolares. E1 hecho de que los conocimientos que se abordan de nuevo deban cambiar de significación no es, en realidad, un descubrimiento para los profesores. Los profesores saben de sobra que existen saberes «oficiales" -nosotros diríamos «institucionalizados»- que se les pueden exigir a los alumnos, y que esos saberes sólo son utilizables por el alumno si establece con ellos una relación privada y si pone en funcionamiento conocimientos de otra naturaleza (personales, contextualizados, temporalizados...). Estas observaciones profesionales sólo adquieren valor en didáctica si determinan un fenómeno de enseñanza explicable por un procesodidáctico o si entran en un fenómeno de ese estilo. Intentemos ilustrar esta deciaración sibilina.

"¿En qué medida el funcionamiento de los saberes "institucionalizados" depende de conocimientos no descontextualizados, enseñados más o menos implícitamente con anterioridad?" (Los conocimientos institucionalizados son los que el maestro enseña explícita y formalmente al alumno, son Ios que constituyen la materia para examen, y el alumno sabe que deben ser aplicados.)

«Comprender», para un niño, es establecer y enlazar, bajo su propia responsabilidad, fenómenos y hechos que tanto el enseñante, como la situación, su lenguaje y los conocimientos aprendidos han dejado «independientes».

Por ejemplo, un niño puede comprender las primeras mediciones con la ayuda del recuento, aprehender las propiedades del orden de los números con la ayuda de la medición, controlar operaciones con la ayuda del orden («eso aumenta, así que no hay que dividir») o de otra operación (multiplicar es añadir un cierto número de veces), comprender el recuento gracias a operaciones (trece es diez más tres) o a la búsqueda de sucesores..., $\mathrm{y}$ todas las relaciones posibles, verdaderas en los enteros, son buenas para dotar de sentido.

No todos estos conocimientos, que el alumno ha entrelazado, personalmente o gracias a la historia de la clase, 
son institucionalizados por la actividad del enseñante, pero, sin duda, algunos de ellos son institucionalizados en el contexto, y esto con toda la razón del mundo. En todo caso, estos conocimientos parecen indispensables para el funcionamiento adecuado de los conocimientos institucionalizados, enseñados por el profesor.

Para el alumno, esas propiedades de los números natura* les son las propiedades de los números en general, de todos los números. Ahora bien, la inmersión del conjunto de los naturales en un superconjunto como los racionales o los decimales, a la vez que hace aparecer propiedades nuevas, hace desaparecer algunas otras; hay propiedades que ya no son verdaderas para todos los números, o incluso que ya no to son para ninguno: multiplicar puede hacer más pequeño, un decimal no tiene sucesor...

El enseñante no puede advertir de manera conveniente al alumno de esta ruptura, ya que, ni la cultura -y en particular la tradición-, ni la ingeniería didáctica han producido todavía los instrumentos necesarios (ejercicios, advertencias, conceptos, observaciones, paradojas...). Esta situación conduce al enseñante a provocar quid pro quos y malentendidos y al alumno a cometer errores. Estas concepciones falsas persisten ya que están ligadas a una cierta manera de comprender las propiedades de los números naturales, y se puede observar los efectos de la ruptura durante numerosos años.

Aún es más importante el mecanismo de este obstáculo: lo que falla no son los conocimientos enseñados -en general los enseñantes subvienen a ese inconveniente intentanto mantenerse en un discurso incomprendido, pero correcto-, lo que falla son los instrumentos personales de comprensión del alumno. El alumno deja de comprender porque lo que debería cambiarse son, precisamente, los medios de lo que él llamaba «comprender» hasta ese momento.

La didáctica no puede aportar solución a un problema como ése mediante simples disposiciones de ingeniería. $\mathrm{La}$ ingeniería puede proponer eventualmente procesos de descontextualización, como los «cambios de marco" de Douady, o situaciones que permiten combatir un conocimiento-obstáculo, como los conflictos sociocognitivos..., pero no puede hacer nada cuando la dificultad viene de que el enseñante desconoce las referencias contextuales del alumno, necesarias para que éste no pierda el fruto de su experiencia con ocasión de nuevas adquisiciones. Es fácil mostrar que las causas de esta imposibilidad de gestionar la memoria de los alumnos gracias a una memoria correcta del sistema son a menudo culturales. El problema debe ser atacado en ese ámbito. La didáctica debe interactuar con la cuitura.

Pero los hechos que acabamos de evocar son los elementos de un fenómeno más amplio que envenena las relaciones entre los padres, los profesores y los alumnos con ocasion de los cambios de clase y de nivel. Un profesor quiere recordar a un alumno que se encuentra en una dificultad los conocimientos que éste necesita; se trata de conocimientos bien institucionalizados: deberían, por tanto, estar disponibles. Pero su uso y su compren. sión dependen de un contexto; si el profesor ignora cuál es ese contexto, la situación está bloqueada. A menudo, el alumno descubre, después de la solución, que conocía perfectamente lo que se le pedia, pero que no habia comprendido la pregunta.

El único medio para el profesor de conocer las circunstancias de la creación de los conocimientos es o bien haber enseñado en persona la noción a ese alumno, o bien disponer de un conjunto de referencias culturales, ya sean éstas efecto de la tradición o de un conocimiento profesional (pero esta especie de «clasicismo" de la didáctica, si consiste en una institucionalización de los problemas, puede resultar una solución peligrosa, que mata la reflexión matemática). Sólo en ese caso puede evocar las situaciones de aprendizaje que él no ha vivido, ya que están fijadas convencionalmente.

Ante las dificultades y las incomprensiones de numerosos alumnos en el uso de conocimientos sencillos, es forzoso constatar el fracaso (del alumno, de la ense. ñanza, del método, del programa...) y pedir que todo (el alumno, la enseñanza...) se centre sobre lo que mejor sabe definir, evaluar y enseñar: los algoritmos; es decir, sobre lo que quizá falla menos, el alumno sabe hacer una división, pero no sabe cuándo hay que hacerla.

Resultado: los responsables se preocupan, los profesores se ponen de acuerdo y, para mejorar el paso de un nivel a otro, la enseñanza ignora y desatiende aún más los conocimientos contextualizados y personales del alumno. El proceso se mantiene a sí mismo hasta el momento en que aparece, para los enseñantes y los alumnos, la necesidad de romper con el psitacismo (la repetición desprovista de sentido). Esto es lo que suce. dió hace algunos años: la noosfera (el conjunto de personas y grupos interesados en la creación y en la comunicación de los saberes de un cierto dominio) invitó a todo el mundo a un esfuerzo de invención, de originalidad y de innovación pedagógica. En consecuencia, se da por supuesto que cada uno ha de ignorar el método personal del otro. El aumento de la variabilidad didáctica espontánea hace entonces que disminuyan las oportunidades, para cada profesor, de articular y de volver a tomar los conocimientos personales de sus alumnos.

La existencia de textos del saber que podían pretender ser universales y definitivos ha facilitado este proceso, pero este proceso ha provocado, de rebote, un aplastamiento de la enseñanza sobre un conocimiento formal...

\subsection{Algunos fenómenos y conceptos de Didáctica}

La didáctica comienza a poner en evidencia los factores de la evolución relativamente caótica de la transposición didáctica, de la que no hablaremos aquí.

El juego del contrato didáctico se acompaña de toda una familia de fenómenos en los que se percibe, a través de los desequilibrios y las correcciones, el efecto de las 
variables del sistema, cuando no las reglas de su evolución.

EI estudio del contrato didáctico ha conducido al descubrimiento de fenómenos como los efectos «Topaze» y «Jourdain», el efecto de deslizamiento metadidáctico, el efecto de analogía..., de los que hablaremos más abajo. Estos efectos pueden inferirse del modelo teórico y pueden observarse tanto en el ámbito de una lección en una clase como en el del conjunto de una comunidad.

El «efecto Topaze» consiste en lo siguiente: la respuesta del alumno está generalmente determinada, poco más o menos, de antemano y el profesor negocia las condiciones en las que se producirá y que la dotarán de sentido. El profesor intenta hacer las cosas de tal manera que ese sentido sea lo más rico y lo más exacto posibie y, para ello, propone las preguntas más abiertas. En caso de fracaso, da información para hacer más fácil la respuesta. Sucede entonces que acaba por aceptar unas condiciones que provocan la respuesta del alumno sin que éste tenga que invertir el menor sentido, como en la primera escena del Topaze de Pagnol: «des moutonsses étai-hunt réunisse... (v).

El «efecto Jourdain» es una forma del efecto Topaze: para evitar un debate de conocimiento con el alumno, y eventualmente la constatación de un fracaso, el profesor acepta reconocer como índice de un saber genuino, o de un modo de actuar auténtico, una producción o un comportamiento del alumno que no es de hecho más que una respuesta que tiene causas triviales $-y$, por tanto, desprovistas de valor e, incluso a veces, de sentido. Ejemplos: Ia escena del «Bourgeois gentilhomme» de Molière en la que el profesor de filosofía revela al señor Jourdain que hace prosa, o la escena en la que habla en cultalatiniparla mientras que Jourdain cree aprender Ia ortografía pronunciando oes y aes.

El deslizamiento metadidáctico alcanzó una gran envergadura y tuvo consecuencias importantes en un pasado reciente; el fenómeno calificado con este nombre consiste en que, cuando un intento de enseñanza fracasa, el profesor se ve conducido a volver a tomar su texto de enseñanza para explicarlo y completarlo. Entonces, el primer intento, que era un medio de enseñanza, deviene objeto de estudio, incluso en ocasiones objeto de enseñanza: Ia forma substituye al fondo. Así, para explicar el lenguaje conjuntista, fundamental, pero en ruptura con el pensamiento natural, los profesores de los años setenta quisieron utilizar, bajo la forma de los famosos diagramas de Venn y otros «globitos», los esquemas que Euler había inventado para Catalina de Rusia en sus «cartas a una princesa de Alemania». Pero, al no ser esta metáfora un buen modelo, y al ayudar la voluntad de vulgarización, hacía falta fabricar sin cesar nuevas convenciones y enseñar el medio de enseñanza como si fuera el objeto đe enseñanza: el lenguaje conjuntista en vez de la lógica, el diagrama en vez del lenguaje formaI, el vocabulario de los diagramas en vez de los dibujos, las convenciones en vez del vocabulario, etc. El deslizamiento metadidáctico escapó al control de la comunidad, provocando magníficos malentendidos a escala planetaria durante más de diez años, sin hablar de las secuelas que experimentamos todavía en la epistemología del público y de los profesores.

Pero existen otros fenómenos de este tipo que dependen del saber al que se apunta y de las circunstancias.

No está claro que las producciones cognitivas hechas para la investigación formen un conjunto de conceptos perfectamente adaptado a su comunicación y a la formación de los alumnos. Existen "puntos ciegos» de la cuItura. Saberes útiles para el desarrollo de un individuo no figuran como nociones científicas en la cultura, y la enseñanza no puede, evidentemente, rellenar esa falta: por ejemplo, la enumeración de colecciones -aspecto muy furtivo de la combinatoria-, o la representación del espacio para la organización de las acciones, de los desplazamientos, de las medidas -sector tragado por la geometría-debate del matemático- o también el pensamiento natural -devorado por la lógica-, etc.

De manera más general, el modo de producción «natural» de la actividad matemática, solicitado sin cesar por los buenos profesores, no puede ser recibido y reconocido verdaderamente en tanto que tal, por tanto, hay que substituirlo por el modo de construccion y el lenguaje que se usa. Si esto no es posible de inmediato, todo el sistema se encuentra ante el problema de primero «memorizar» conocimientos transitorios, de estatuto incierto, no reconocidos por la ciencia, y luego hacerlos evolucionar, sin poder expresarlos ni reconocerlos.

El esfuerzo consentido para obtener saberes, independientes de las situaciones en los que funcionan (descontextualización), se paga en pérdida de sentido y de operatividad en el momento de la enseñanza. El restablecimiento de situaciones (recontextualización) inteligibles se paga en deslizamientos de sentido (transposición didáctica). La retransformación en saberes del alumno o en saberes culturales vuelve a iniciar el proceso y agrava los riesgos de deriva. La didáctica es el medio de gestionar estas transformaciones $y$, en primer lugar, de comprender sus leyes.

Volvamos a la gestión de las expresiones aligebraicas por los alumnos. Yves Chevallard ha mostrado el fenómeno de conservación ostensiva de la información: un contrato didáctico implícito encarga al alumno que «conserve» Ia información que se le confía; desde ese punto de vista, en la expresión « $3 . x=0 »$ el 3 muestra algo: es diferente de 4; debe, por tanto, permanecer presente a través de las transformaciones matemáticas. Deducir de la expresión anterior « $\mathrm{x}=0$ » contradiría este contrato, de manera que el alumno un poco distraído transforma el resultado en $\ll \mathrm{x}=1 / 3 »$ o en $« \mathrm{x}=-3$ ».

El inventario de los fenómenos ligados al contrato diđáctico aún está en plena expansión.

Sin embargo, mostrar y estudiar fenómenos es una cosa, actuar sobre ellos, es otra. Trabajos de la coherencia de los de M. Artigue son aún rarísimos: después de haber estudiado la reproductibilidad de las situaciones diđácticas, ella observó la obsolescencia de las situaciones utilizadas para la enseñanza de las ecuaciones diferenciales y ha comenzado a proponer soluciones. 


\section{CONCLUSIONES}

La didáctica puede, en un cierto plazo, ayudar al profesor a modificar su estatuto, su formación y sus relaciones con la sociedad:

-Actuando directamente sobre la consideración social de los conocimientos que utiliza.

-Actuando sobre los conocimientos de sus colegas pro. fesionales, y sobre los de los padres y el público en general.

-Desarrollando posibilidades mejores, para el público y para los ciudadanos, de utilizar la enseñanza de manera más satisfactoria para ellos.

-Dando mejores posibilidades a los poderes públicos o privados de gestionar la enseñanza mediante medios más apropiados.

\section{Notas}

(1) Presenté y estudié está lección en Brousseau (I981). Ha sido evocada en Clapponi (1985) y Pluvinage (1986). Morin (1988) da de forma detallada los comportamientos de los alumnos observados en el curso de las repeticiones que ella ha organizado de esa lección.

(2) El conjunto del proceso se describe de manera detallada en Brousseau, N. y Brousseau, G. (1987).

(3) Esta lección es solamente la introducción a un proceso que debe llegar hasta su término, para que los alumnos puedan encontrarse con todos los aspectos del empleo de las aplicacio. nes racionales y decimales, y dominarlos. Aislada, colocada en un contex to desordenado, o gestionada sin rigor, esta lección corre el riesgo de funcionar meramente como un ejercicio de aplicación o como un soporte metaforico del discurso enseriado. Pero esta técnica es bastante robusta: incluso mal analizada y mal empleada, todavía guarda un atractivo cierto.

\section{REFERENCIAS BIBLIOGRÁFICAS}

BROUSSEAU, G., 1981. Problèmes de didactique des déci. maux, Recherches en Didactique des Mathématiques, Vol. 2, pp. $37-127$.

BROUSSEAU, N. y BROUSSEAU, G., 1987. Rationnels et décimaux dans la scolarité obligatoire. IREM de Bordeaux.

CENTENO, J., 1988. Números decimales. (Síntesis: Madrid).

CHEVALLARD, Y., 1989. Le passage de l'ariłhmétique à l'algèbre dans l'enseignement des mathématiques au collè. ge, Petit $X, 19$, pp. $43 \cdot 72$.
Esta parte de las aportaciones de la didáctica no está, ciertamente, lista para ser realizada, ya que exige una evolución considerable de las estructuras escolares y de las mentalidades, pero todo me permite pensar que ése es su papel social y que progresamos en esa dirección.

La vocación đe la didáctica se opone a las intervenciones atronadoras. A menudo, la explicación de un fracaso o de una dificultad permite desculpabilizar al enseñante y al alumno y orientarles hacia actitudes más positivas. En medicina, Ia atribucion de la tuberculosis a un microbio no permitió de inmediato vencer la enfermedad, pero permitio por fín desculpabilizar a los enfermos, sospechosos durante mucho tiempo de haber ofendido a la naturaleza de alguna manera y de ser castigados por ello; accesoriamente, la medicina sugirió que un cierto tipo de higiene podría prevenir muchos casos.

\section{Notas det traductor}

(I) 'Falsable', o 'falsar', es un neologismo introducito por los traductores de Lakatos al castellano.

(II) Siglas correspondientes a lo que en la enseñanza francesa se denomina "Cour Moyen deuxième année», que equivale a $5^{\circ}$ de EGB.

(III) Equivalente $6^{8}$ de EGB.

(IV) Juego de palabras: 'maitre' significa 'amo' y 'maestro'; en el original francés se dice entre paréntesis «c'est bien le mot», «es la palabra adecuada».

(V) En la obra de Marcel Pagnol, Topaze es un profesor al que el director del colegio privado para niños ricos en que trabaja le exige que obtenga mejores resultados. Agobiado por esta exigencia que pone en peligro su puesto de trabajo, para conseguir que sus alumnos no tengan faltas en los dictados, pronuncia con énfasis todas las letras que, como las eses finales de 'moutons' o 'réunis', no se pronuncian en francés.

CLAPPONI, Ph., 1985. Activité... découpes, Petit $X, 9$, pp. $63-$ 65.

PLUVINAGE, F. y RAUSCHER, J. C., 1986. La géometrie construite mise à l'essai, Petit $X, 11, \mathrm{pp} .5-36$.

MORIN, C., 1988. Le puzzle (d'après une idée de G. Brousseau), Petit $X, 17$, pp. 49-56. 